\title{
Characterization of Nickel Based Superalloys Processed Through Direct Metal Laser Sintering Technique of Additive Manufacturing
}

\author{
Yaakov Idell ${ }^{1}$, Carelyn Campbell ${ }^{1}$, Lyle Levine ${ }^{1}$, Fan Zhang ${ }^{1}$, G. Olson ${ }^{2}$, D. Snyder ${ }^{2}$ \\ 1. Materials Science and Engineering Division, National Institute of Technology and Standards, \\ Gaithersburg, MD, USA \\ 2. QuesTek Innovations LLC, Evanston, IL, USA
}

Ni-based superalloys are widely used for their excellent mechanical properties, particularly in the field of high temperature applications, through the control of $\gamma$ and $\gamma^{\prime}$ phase ratios [1]. Due to the advances in technology, additive manufacturing of Ni-based superalloys will allow direct production of complex shaped components based on 3-D computer aided drawings. The aerospace industry is interested in exploiting this technology to reduce time and cost for production of complex parts; however, the effects resulting from repeated cycles of rapid heating, melting, cooling, and solidification on the microstructure-property relationships are not well understood. Traditionally, microstructural studies of Ni-based superalloys are limited to the transmission electron microscope (TEM) due to the small precipitate phases $\left(\gamma^{\prime}, \gamma^{\prime}\right.$, and $\delta$ phases); however, this characterization technique will not provide statistically significant data sets. The most extensively used X-ray technique for statistically significant characterization of bulk microstructure is small angle X-ray scattering (SAXS). In SAXS, the elastic scattering from low scatter angles within a material can reveal information regarding parameters such as the size, shape, volume, and information regarding scatters for both ordered or partially ordered scattering through small changes in the electron density induced by the long-range elastic dilation field, density changes in the dislocation core, and the nonlinear part of the elastic field [2]. Information delivered using SAXS can resolve feature sizes from $1 \mathrm{~nm}$ to $100 \mathrm{~nm}$, which is not a useful technique when dealing with precipitates in the ultrafine and submicron grain size regime. For resolving microstructures in these size regimes, ultra-small angle X-ray scattering (USAXS) will need to be utilized as the smaller scatter angle is sensitive to microstructures as large as $30 \mu \mathrm{m}$ through a BonseHart double crystal configuration with a silicon monochromator, attenuators, filters, and a variable slit [3].

Here we report and discuss the evolution of the microstructure-property relationships of Allvac 718Plus, a Ni-based superalloy, that have been built using direct metal laser sintering and its response to annealing at $1066{ }^{\circ} \mathrm{C}$. In-situ annealing experiments using a combined USAXS/SAXS setup at the Advanced Photon Source in Argonne National Laboratory is shown in Figure 1a and 1b were performed, which is sensitive to feature sizes over a three decade range from just below $1 \mathrm{~nm}$ to just above $30 \mu \mathrm{m}$ [4]. Complementary ex-situ experiments for the as-built and annealed microstructures are characterized through scanning and transmission electron microscopy, electron back scattering diffraction, X-ray diffraction, and synchrotron microbeam diffraction to determine the residual stress distributions, porosity, phase fractions, and compositional differences in an effort to develop the microstructureproperty relationships. These results are compared with multicomponent diffusion simulations and FEM simulations that predict the phase fraction, composition, and residual stress as functions of time and temperature.

Figure 1c shows an example of 1D-collimated USAXS/SAXS during an in-situ annealing experiment from room temperature to $1066{ }^{\circ} \mathrm{C}$. The 718 Plus as built morphology shows an microstructure with 
significantly elongated grains (aspect ratio of $\approx 6.5$ ) with a dendritic solidification structure and possible $\delta$ precipitates present in Nb-rich interdendritic regions, a strong $\{200\}$ cube texture, and tensile strength of $\approx 1270 \mathrm{MPa}$. Scheil solidification simulation and thermodynamic modeling indicate that regions of high compositional imbalance, such as the observed high concentrations of $\mathrm{Nb}$ in interdendritic regions, can lead to the formation of the undesirable carbides and $\delta$ phase. These results are similar to a previous study of Inconel 718, which is a Ni-based superalloy with a similar but slightly different composition than 718Plus, as-built by the additive manufacturing technique of electron beam melting [5]. Additionally, the processing technique introduces significant amounts of local tensile residual stress with local variations up to $\approx 1 \mathrm{GPa}$. Upon annealing, the microstructure is altered to an equi-axed morphology with no subgrains or dendritic structure, $\delta$ precipitates along grain boundaries, and local tensile residual stress, and a tensile strength of $\approx 720 \mathrm{MPa}$. By observing the size, shape, and volume evolution of the precipitates through the use of the USAXS/SAXS instrument, it is evident that the $\delta$ precipitates are growing with time. The significantly increased local $\mathrm{Nb}$ concentration from the DMLS processing segregation in turn causes $\delta$ precipitate dissolution temperature to increase as well [6] allowing $\delta$ precipitate growth. The USAXS/SAXS data will aid in selecting an optimal solutionizing heat treatment time to balancing stress relief, homogenized composition, and an equi-axed non-dendritic microstructure while minimizing $\delta$ precipitate and matrix grain size.

[1] D.M. Shah, D.N. Duhl, Superalloys, (1984) 105-114.

[2] L. Levine, G. Long, Diffuse Scattering and the Fundamental Properties of Materials, (2009) 345.

[3] U. Bonse, M. Hart, Applied Physics Letters, 7 (1965) 238-240.

[4] J. Ilavsky, et al, Journal of Applied Crystallography, 42 (2009) 469-479.

[5] A. Strondl, et al, Materials Science and Technology, 27.5 (2011) 876-883.

[6] R.E. Schafrik, et al, TMS, (2001) 1-11.

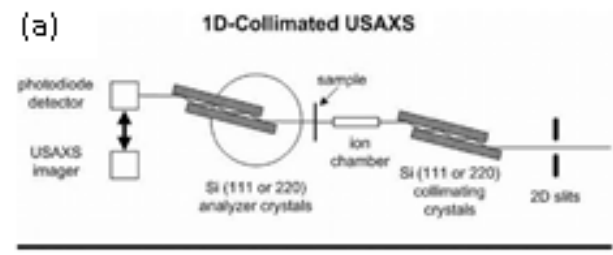

(b)

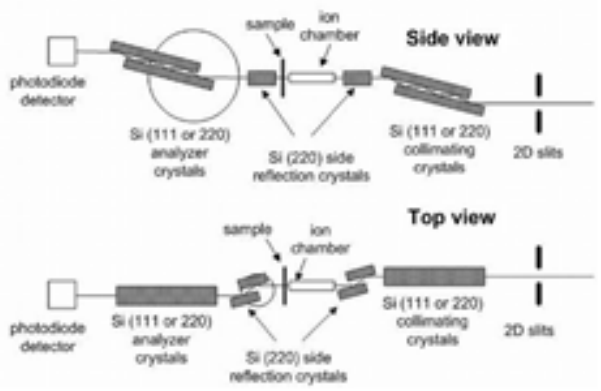

(c)

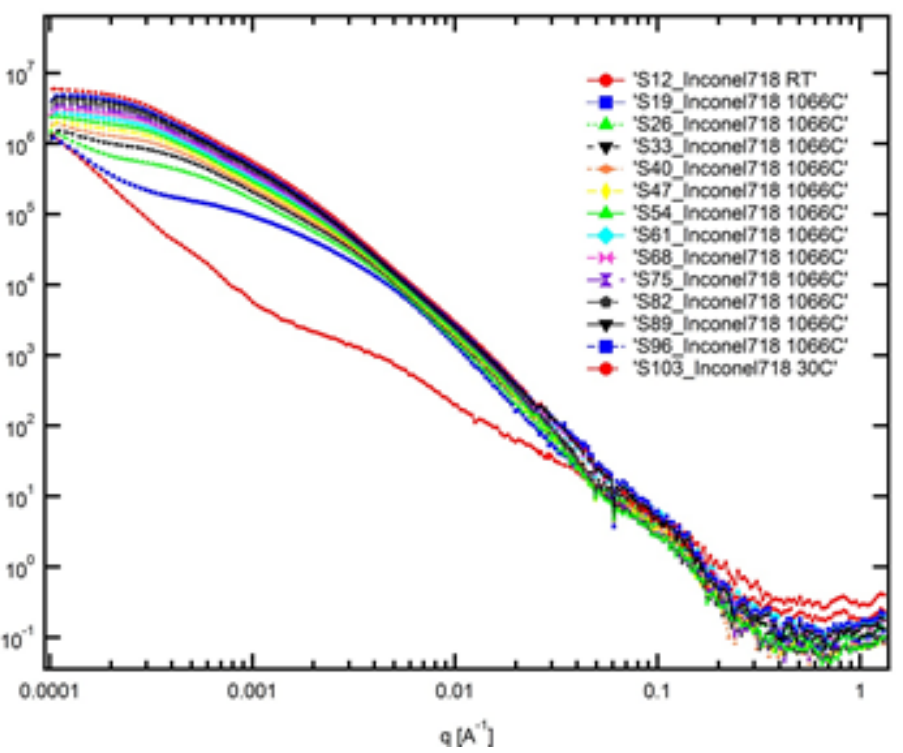

Figure 1: Schematic of the APS USXS instrument in (a) one-dimensional collimated or USAXS imaging configuration, and (b) two-dimensional collimated configuration; (c) example of statistically relevant USAXS/SAXS data set illustrating microstructural changes during in-situ annealing experiment. 